Pacific Journal of Mathematics

THE LARGEST DIGIT IN THE CONTINUED FRACTION 


\section{THE LARGEST DIGIT \\ IN THE CONTINUED FRACTION EXPANSION OF A RATIONAL NUMBER}

\section{Douglas Hensley}

The finite continued fraction sequence of a reduced fraction $a / b$, with $0 \leq a<b$, is the sequence $d=(d(1), d(2), \ldots, d(r))$ of positive integers such that $d(r)>1$, and

$$
a / b=1 /(d(1)+1 /(d(2)+\cdots+1 / d(r))) .
$$

In the standard terminology of continued fractions, this is written as $[0 ; d(1), d(2), \ldots, d(r)]$, which we abbreviate here to $[d(1)$, $d(2), \ldots, d(r)]$. Thus $[1,4,2]=1 /(1+1 /(4+1 / 2))=9 / 11$. The empty sequence corresponds to $0 / 1$. For any other fraction, there will be $r \geq 1$ digits (also known as partial quotients) $d(j)$ in this expansion $(1 \leq j \leq r)$. The largest of these we call $D(a / b)$ or $D(a, b)$. Thus $D(9 / 11)=D(9,11)=4$. The aim of this work is to elucidate the distribution of $D(a, b)$. Put informally, the main result is that $\operatorname{Prob}[D(a, b) \leq \alpha \log b] \approx \exp \left(-12 / \alpha \pi^{2}\right)$. More precisely, it is shown that for all $\varepsilon>0$, and uniformly in $\alpha>\varepsilon$ as $x \rightarrow \infty$,

$$
\begin{aligned}
\#\{(a, b): 0 & \leq a<b \leq x, \operatorname{gcd}(a, b)=1, \text { and } D(a, b) \leq \alpha \log x\} \\
& \approx\left(3 / \pi^{2}\right) x^{2} \exp \left(-12 / \alpha \pi^{2}\right) .
\end{aligned}
$$

The question of how often there are exactly $M$ digits exceeding $\alpha \log b$ in the continued fraction expansion of a reduced fraction $a / b$ with $0 \leq a<b \leq x$ is also touched on. Evidence points to the estimate

$$
\left(3 / \pi^{2}\right) x^{2}(M !)^{-1}\left(12 / \alpha \pi^{2}\right)^{M} \exp \left(-12 / \alpha \pi^{2}\right)
$$

for the number of such fractions.

Previous work in a similar vein includes a result of Galambos $[4,5]$ concerning the distribution of the continued fraction partial quotients (digits) of a randomly chosen real number in the interval $(0,1)$. Corresponding to any irrational $\xi$ in $(0,1)$ there is a unique sequence $d_{\xi}=d=(d(1), d(2), \ldots)$ of positive integers such that

$$
\xi=[d]=[d(1), d(2), \ldots]=1 /(d(1)+1 /(d(2)+\cdots)) .
$$

Galambos found that if $X$ is a random variable uniformly distributed on $[0,1]$ (in the statement of his result the random variable has the Gauss-Kuzmin distribution, but that was just a convenience), then

$$
\lim _{r \rightarrow \infty} \operatorname{Prob}\left(\max _{k \leq r} d_{X}(k)<\alpha r\right)=e^{-1 / \alpha \log 2} .
$$


There is also a literature concerning the distribution of pairs $(a, b)$ for which, in the finite continued fraction expansion $d=d_{a / b}$ of $a / b$, all of the $d(j)$ are bounded by some fixed $N$. It is known $[2,3,8]$ that for each $N \geq 2$ there exists a real number $H(N), 0<H(N)<1$, such that the number of pairs $(a, b)$ for which $b \leq x$ is on the order of $x^{2 H(N)}$, uniformly in $N$ as $x \rightarrow \infty$. For each fixed $N$, there is also [9] a constant $C(N)>0$ such that this pair count is $\approx C(N) x^{2 H(N)}$, but it is not known how fast the convergence to this asymptotic behavior is, or whether it is uniform in $N$. There is no evident reason to suspect that it would not be uniform, but in any event numerical evidence suggests that $x$ must be fairly large before the asymptotic trend takes hold. Recently, the author also showed [10] that

$$
\lim _{n \rightarrow \infty} N(1-H(N))=6 / \pi^{2} .
$$

As usual, $\Phi(x)$ denotes $\sum_{n \leq x} \phi(n)=\#\{(a, b): 0 \leq a<b \leq x$ and $\operatorname{gcd}(a, b)=1\}$, so that

$$
\Phi(x) \approx\left(3 / \pi^{2}\right) x^{2} \text { as } x \rightarrow \infty .
$$

Now let

$$
\begin{aligned}
\Phi(x, \alpha):=\#\{(a, b): 0 \leq a<b \leq x, & \operatorname{gcd}(a, b)=1, \\
& \text { and } D(a, b) \leq \alpha \log x\} .
\end{aligned}
$$

From the results mentioned above, it follows that there exists $C>0$ such that for all sufficiently large $x$,

$$
(1 / C) x^{2} e^{-12 / \alpha \pi^{2}} \leq \Phi(x, \alpha) \leq C x^{2} e^{-12 / \alpha \pi^{2}}
$$

whenever $\alpha \log x$ is an integer $\geq 2$. In view of the results just mentioned, our main result below fits in nicely:

THEOREM 1. Uniformly in $\alpha \geq 4 / \log \log x$ as $x \rightarrow \infty$, $\Phi(x, \alpha)=\left(3 / \pi^{2}\right) x^{2} e^{-12 / \alpha \pi^{2}}\left(1+O\left(\left(\alpha^{-2}+1\right) e^{24 / \pi^{2} \alpha} \log \log x / \log x\right)\right)$ as $x \rightarrow \infty$.

The result can also be put in a form which refers to the Diophantine approximation properties of $a / b$ rather than to its continued fraction expansion. Let

$$
\delta(a, b):=\min _{1 \leq k<b}\|k a / b\|,
$$


where $\|u\|$ denotes the distance from $u$ to the nearest integer. Let

(5) $F(x, \alpha):=\#\{(a, b): 0 \leq a<b \leq x, \operatorname{gcd}(a, b)=1$,

and $\delta(a, b)>1 / \alpha \log x\}$.

Then for fixed $\alpha>0$, as $x \rightarrow \infty$,

$$
F(x, \alpha) \approx\left(3 / \pi^{2}\right) x^{2} e^{-12 / \alpha \pi^{2}} .
$$

The basic idea of the proof is to count $\Phi(x, \alpha)$ by inclusion and exclusion, throwing out all fractions with at least one digit too largeonce for each such digit - then restoring those with at least two-once for each such pair of digits-and so on. Term by term, these counts are asymptotic to the corresponding term in the identity

$$
\left(3 / \pi^{2}\right) x^{2} e^{-12 / \alpha \pi^{2}}=\left(3 / \pi^{2}\right) x^{2} \sum_{j=0}^{\infty}\left(-12 / \pi^{2} \alpha\right)^{j} /(j !) .
$$

REMARK. A more sophisticated version of inclusion and exclusion yields an asymptotic estimate of the number of fractions with exactly $M$ digits $\geq \alpha \log x$, and denominator $\leq x$. Let $\mu_{M}: \mathbb{N} \rightarrow \mathbb{Z}$ satisfy

$$
\sum_{k=0}^{n}\left(\begin{array}{l}
n \\
k
\end{array}\right) \mu_{M}(k)=\{1 \text { if } n \leq M, 0 \text { if not }\} .
$$

This defines $\mu_{M}$ recursively, and it is not hard to see that $\mu_{M}(0)=1$,

$$
\begin{gathered}
\mu_{M}(j)=0 \text { if } 1 \leq j \leq M, \quad \text { and } \\
\mu_{M}(j)=(-1)^{(j-M)}\left(\begin{array}{c}
j-1 \\
M
\end{array}\right) \quad \text { for } j>M .
\end{gathered}
$$

Following the proof given here for the case $M=0$, but with $\mu_{M}$ in place of $(-1)^{j}$, leads to a main term of

$$
\left(3 x^{2} / \pi^{2}\right)(1 / M !)\left(12 / \pi^{2} \alpha\right)^{M} e^{-\left(12 / \pi^{2} \alpha\right)} .
$$

2. Inclusion and exclusion. Let $V_{r}:=\left\{v:\{1,2, \ldots, r\} \rightarrow \mathbb{Z}^{+}\right\}$be the set of all sequences of $r$ positive integers, and let $V=\bigcup_{r=0}^{\infty} V_{r}$. For $v \in V_{r}$, let lex $(v)=r$, the lexicographic length of $v$.

Let $a_{0}(v)=0, b_{0}(v)=1, a_{-1}(v)=1$, and $b_{-1}(v)=0$. For $1 \leq i \leq r$ we define $a_{i}(v)$ and $b_{i}(v)$ recursively by the conditions

$$
\begin{aligned}
& a_{i}(v)=d_{i} a_{i-1}(v)+a_{i-2}(v), \\
& b_{i}(v)=d_{i} b_{i-1}(v)+b_{i-2}(v),
\end{aligned}
$$


where $d_{i}=v(i)$ is the $i$ th entry in the sequence $v$. Let

$$
\langle v\rangle:=b_{\operatorname{lex}(v)}(v)=b_{r}(v),
$$

say, let $[v]:=a_{r}(v) / b_{r}(v)$, and $\{v\}:=b_{r-1}(v) / b_{r}(v)$. By convention, if $v$ is the empty sequence $(r=\operatorname{lex}(v)=0)$ then $\langle v\rangle=1$ and $[v]=\{v\}=0$.

There is a two-to-one correspondence $T$ between $V$ and $\{(a, b)$ : $0 \leq a<b$ and $\operatorname{gcd}(a, b)=1\}$. In one direction, we map $v \stackrel{T}{\rightarrow}\left(a_{r}, b_{r}\right)$ where $r=\operatorname{lex}(v)$. In the other direction, $\operatorname{cfx}(a, b)$ is defined as that one of the two $v$, mapped by $T$ back to $(a, b)$, for which the last entry $v(r)$ is greater than one. The other, call it $\tilde{v}$, is obtained by replacing $v(r)$ with $v(r)-1$, and appending 1 as $\tilde{v}(r+1)$. Thus

(9) $\#\{v \in V:\langle v\rangle \leq x$ and $v(i) \leq \alpha \log x-1$ for $1 \leq i \leq \operatorname{lex}(v)\}$

$$
\leq 2 \Phi(x, \alpha) \leq \#\{v \in V:\langle v\rangle \leq x \text { and } v(i) \leq \alpha \log x
$$

for $1 \leq i \leq \operatorname{lex}(v)\}$.

In (9) we don't get equality because there are some $v \in V$ such that $v(r)-1 \leq \alpha \log x<v(r)$. Given two sequences $u, w \in V$, we write $u w$ for their concatenation. That is, $u w$ denotes the sequence $v$ such that $v(j)=u(j)$ for $j \leq \operatorname{lex}(u), \operatorname{lex}(v)=\operatorname{lex}(u)+\operatorname{lex}(w)$, and $v(j)=w(j-\operatorname{lex}(u))$ for $\operatorname{lex}(u)<j \leq \operatorname{lex}(u)+\operatorname{lex}(w)$. With this notation, a well-known identity reads

$$
\langle u w\rangle=\langle u\rangle\langle w\rangle(1+\{u\}[w]) .
$$

Now if $d_{j}=u(j)>N$, (where in the subsequent application, $N=$ $[\alpha \log x])$, then $\{u\}=b_{j-1}(u) /\left(d_{j} b_{j-1}(u)+b_{j-2}(u)\right)<1 / N$, so that for $u \in V_{j}$ with $u(j)>N$, and $w \in V$,

$$
\langle u\rangle\langle w\rangle \leq\langle u w\rangle \leq(1+1 / N)\langle u\rangle\langle w\rangle .
$$

This gives us a way to estimate, for $1 \leq l \leq L$ say, the number of constructions of the form

$$
v=u_{1} k_{1} u_{2} k_{2} \cdots u_{l} k_{l} u_{l+1}, \quad \text { with } u_{1}, u_{2}, \ldots, u_{l+1} \in V,
$$

$k_{1}, k_{2}, \ldots, k_{l} \in V(1)$ or $\mathbb{Z}^{+}$(which we equate by a sleight of notation), with all $k_{i}>N$, and with $\langle v\rangle \leq x$. Note that since $V$ includes the empty sequence, there need not be any genuine interposition between consecutive $k_{i}$. Note also that the same sequence, if more than $l$ of the $v(i)$ are greater than $N$, can be expressed in the above form in more than one way. 
Our inclusion and exclusion argument is based on counting representations of $v$ of the above form. For every integer $l \geq 0$, and every $v \in V$, let $\sigma(v, l, N)$ denote the number of ways in which $v$ can be written as $u_{1} k_{1} \cdots u_{l} k_{l} u_{l+1}$, with all $k_{i}>N$. Then

$$
\sum_{l=0}^{\infty}(-1)^{l} \sigma(v, l, N)= \begin{cases}1 & \text { if all } v(i) \leq N, \\ 0 & \text { if any } v(i)>N,\end{cases}
$$

and $\sum_{l=0}^{K}(-1)^{l} \sigma(v, l, N)$ alternates about this, being $\geq\{1$ resp. 0$\}$ for $K$ even, and $\leq\{1$ resp 0$\}$ for $K$ odd. Now let

$$
\begin{array}{r}
\Phi^{-}(x, \alpha):=\frac{1}{2} \#\{v \in V:\langle v\rangle \leq x \text { and } v(i) \leq \alpha \log x-1 \\
\text { for } 1 \leq i \leq \operatorname{lex}(v)\},
\end{array}
$$

and

$$
\begin{aligned}
& \Phi^{+}(x, \alpha):=\frac{1}{2} \#\{v \in V:\langle v\rangle \leq x \text { and } v(i) \leq \alpha \log x \\
& \text { for } 1 \leq i \leq \operatorname{lex}(v)\} .
\end{aligned}
$$

Then with $N=[\alpha \log x]$ or $[\alpha \log x]-1$ respectively,

$$
\begin{aligned}
\Phi^{ \pm}(x, \alpha)= & \frac{1}{2} \sum_{v \in V} \chi(\langle v\rangle \leq x) \sum_{l=0}^{\infty}(-1)^{l} \sigma(v, l, N) \\
= & \frac{1}{2} \sum_{l=0}^{\infty}(-1)^{l} \sum_{u_{1} \in V} \sum_{k_{1}>N} \sum_{u_{2} \in V} \\
& \cdots \sum_{k_{l}>N} \sum_{u_{l+1} \in V} \chi\left(\left\langle u_{1} k_{1} \cdots u_{l} k_{l} u_{l+1}\right\rangle \leq x\right) .
\end{aligned}
$$

Now let $W_{l}(x, N)$ denote the number of pairs $\left(\left(u_{1}, u_{2}, \ldots, u_{l+1}\right)\right.$, $\left.\left(k_{1}, k_{2}, \ldots, k_{l}\right)\right)$ where the $u_{i} \in V$ and the $k_{i}>N$, and such that $\left\langle u_{1} k_{1} u_{2} k_{2} \cdots u_{l} k_{l} u_{l+1}\right\rangle \leq x$. Let $W_{l}^{\prime}(x, N)$ denote the number of such pairs for which $\prod_{i=1}^{l} k_{i} \prod_{j=1}^{l+1}\left\langle u_{j}\right\rangle \leq x$. Then from (11), we see that

$$
W_{l}^{\prime}\left((1+1 / N)^{-2 l} x, N\right) \leq W_{l}(x, N) \leq W_{l}^{\prime}(x, n) .
$$

But

$$
\text { (15) } \begin{aligned}
W_{l}^{\prime}(x, N)= & 2^{l+1} \sum_{k_{1}>N} \sum_{b_{1}=1}^{\infty} \sum_{k_{2}>N} \sum_{b_{2}=1}^{\infty} \\
& \cdots \sum_{k_{l}>N} \sum_{b_{l+1}=1}^{\infty} \prod_{i=1}^{l+1} \phi\left(b_{i}\right) \chi\left(\prod_{i=1}^{l+1} b_{i} \prod_{j=1}^{l} k_{j} \leq x\right) .
\end{aligned}
$$


In view of this, it is natural to seek estimates for

$$
A_{m}(y):=\sum_{b \in V_{m}} \prod_{i=1}^{m} \phi\left(b_{i}\right) \chi\left(\prod_{i=1}^{m} b_{i} \leq y\right)
$$

and then to apply them with $y=\left(x / k_{1} \cdots k_{l}\right)$ and $m=l+1$. Another way to write (15), using the definition above, is

$$
\begin{aligned}
& 2^{m} A_{m}(y)=\#\left\{\left(u_{1}, u_{2}, \ldots, u_{m}\right): u_{i} \in V\right. \\
& \left.\qquad \text { for } 1 \leq i \leq m \text { and } \prod_{i=1}^{m}\left\langle u_{i}\right\rangle \leq y\right\} .
\end{aligned}
$$

Once we have suitable estimates for $A_{m}(y)$, we will use (13), together with (17) below:

$$
\begin{aligned}
& \sum_{k_{1}=N+1}^{\infty} \ldots \sum_{k_{l}=N+1}^{\infty} A_{l+1}\left((1+1 / N)^{-2 l} x / k_{1} k_{2} \cdots k_{l}\right) \\
& \leq W_{l}(x, N) \leq 2^{l+1} \sum_{k_{1}=N+1}^{\infty} \ldots \sum_{k_{l}=N+1}^{\infty} A_{l+1}\left(x / k_{1} k_{2} \cdots k_{l}\right) .
\end{aligned}
$$

(There are only finitely many nonzero terms in the sums of (17), as $A_{m}(y)=0$ for $y<1$.) But (13) can now be written as

$$
\Phi^{ \pm}(x, \alpha)=\frac{1}{2} \sum_{l=0}^{\infty}(-1)^{l} W_{l}\left(x, N^{ \pm}\right),
$$

where $N=[\alpha \log x]$ or $[\alpha \log x]-1$ for $\Phi^{+}$or $\Phi^{-}$respectively.

We are now in a position to sketch out the proof of Theorem 1 . First we obtain an estimate of the form (with $\lambda=6 / \pi^{2}$ )

$$
A_{m}(y) \approx \frac{1}{2} y^{2} \lambda^{m}(\log y)^{m-1} /(m-1) !,
$$

by a study of the Dirichlet series

$$
\int_{1}^{\infty} t^{-s} d A_{k}(t)=\left(\sum_{n=1}^{\infty} n^{-s} \phi(n)\right)^{k}=(\zeta(s-1) / \zeta(s))^{k}
$$

Next, we estimate the sums of (17), which from (19) are given approximately by

(21) $2^{l+1} \sum_{\substack{k_{1}=N+1 \\ k_{1} k_{2} \cdots k_{l} \leq x}}^{\infty} \cdots \sum_{k_{l}=N+1}^{\infty} \frac{1}{2} x^{2} \lambda^{l+1}(l !)^{-1} \prod_{i=1}^{l} k_{i}^{-2}\left(\log \left(x / k_{1} \cdots k_{l}\right)\right)^{l}$, 
as

$$
\text { (22) } \begin{aligned}
& \frac{1}{2} x^{2}(2 \lambda)^{l+1}(l !)^{-1} \\
& \quad \times \iint \cdots \int_{R}\left(\log x-\sum_{i=1}^{l} \log t_{i}\right)^{l} t_{1}^{-2} \cdots t_{l}^{-2} d t_{l} \cdots d t_{1},
\end{aligned}
$$

where $R=\left\{\left(t_{1}, \ldots, t_{l}\right): t_{1} \geq N, \ldots, t_{l} \geq N\right.$ and $\left.t_{1} t_{2} \cdots t_{l} \leq x\right\}$. Calculus and simplifying estimates then reduce the integral expression above to about $\frac{1}{2} x^{2}(2 \lambda)^{l+1}(l !)^{-1} \alpha^{-l}$. Finally, from (18) we expect to find that $2 \Phi(x, \alpha)$ is given approximately by

$$
\begin{aligned}
& x^{2} \lambda-\frac{1}{2} x^{2} \sum_{l=1}^{\infty}(-2 \lambda)^{l+1} \alpha^{-l} / l ! \\
& =\lambda x^{2} \sum_{l=0}^{\infty}(-2 \lambda / \alpha)^{l} / l !=\lambda x^{2} e^{-2 \lambda / \alpha},
\end{aligned}
$$

which is roughly what is claimed in Theorem 1.

In $\S 3$ we give details for the estimation of $A_{m}(y)$. In $\S 4$ we give details of the resulting estimates of $W_{l}(x, N)$, and tie it all together.

3. Bounds for $A_{m}(y)$. Recall that $A_{m}(y)=\sum_{b_{1} b_{2} \cdots b_{m} \leq y} \prod_{1}^{m} \phi\left(b_{i}\right)$.

LEMMA 1. There is a positive absolute constant $C$ such that for $1 \leq$ $k \leq C \sqrt{\log y}, A_{k}(y)=\left(\frac{1}{2} \lambda^{k} \log ^{k-1} y /(k-1) !\right) y^{2}\left(1+O\left(k^{2} / \log y\right)\right)$.

Proof. First we note that if Lemma 1 holds for integer $y \geq 3$, then it holds for real $y \geq 3$ as well. Also, the case $k=1$ is the well-known result $\sum_{n \leq y} \phi(n)=\frac{1}{2} \lambda y^{2}(1+O(1 / \log y))$. Now let

$$
\begin{aligned}
f(s, k) & =\left(\sum_{n=1}^{\infty} n^{-s} \phi(n)\right)^{k} \\
& =(\zeta(s-1) / \zeta(s))^{k}=\sum_{n=1}^{\infty} a(k, n) n^{-s}, \text { say. }
\end{aligned}
$$

The series representations of $f(s, k)$ are absolutely convergent, uniformly in $\operatorname{Re}(s) \geq c$ for each $c>2$, and the zeta function representation provides the analytic continuation into the domain $\operatorname{Re}(s) \geq 4 / 3$, apart from a single pole of order $k$ at $s=2$.

For the analysis ahead, it will be more convenient to first study $B_{k}(y):=\sum_{n=1}^{y} A_{k}(n)$, and to establish (for some fixed $C, 0<C<$ $1)$, the following lemma. 
LEMMA 2. Uniformly in

$$
\begin{gathered}
k \leq C \sqrt{\log y}, \quad \text { as } y \rightarrow \infty \\
B_{k}(y)=\left((1 / 6) y^{3} \lambda^{k}(\log y)^{k-1} /(k-1) !\right)\left(1+O\left(k^{2} / \log y\right)\right) .
\end{gathered}
$$

Before proving Lemma 2, we show how Lemma 1 follows from this secondary lemma.

Since $A_{k}(n)$ is increasing in $n$, for any integer $m, 0<m \leq y$, we have

$$
m A_{k}(y) \leq B_{k}(y+m)-B_{k}(y) .
$$

Now from Lemma 2,

$$
\begin{aligned}
B_{k}(y+ & m)-B_{k}(y) \\
= & \left((1 / 6) \lambda^{k} /(k-1) !\right)\left((y+m)^{3} \log ^{k}(y+m)-y^{3} \log ^{k} y\right) \\
& +O\left(k^{2} y^{3} \lambda^{k} \log ^{k-2} y /(k-1) !\right) .
\end{aligned}
$$

Taking $m=[k y / \log y]$, and bearing in mind that $k \leq C \sqrt{\log y}$, this gives

$$
\begin{aligned}
B_{k}(y+m)-B_{k}(y) \\
\quad=\left(\frac{1}{2} m \lambda^{k} y^{2} \log ^{k-1} y /(k-1) !\right)\left(1+O\left(k^{2} / \log y\right)\right) .
\end{aligned}
$$

Thus

$$
A_{k}(y) \leq\left(\frac{1}{2} \lambda^{k} y^{2} \log ^{k-1} y /(k-1) !\right)\left(1+O\left(k^{2} / \log y\right)\right) .
$$

A similar calculation, starting from $A_{k}(y) \geq B_{k}(y)-B_{k}(y-m)$, gives a reversed version of (28). Taken together, these constitute the conclusion of Lemma 1.

We now turn to the proof of Lemma 2. By Perron's formula, for $c>2$ we have

$$
B_{k}(y)=\frac{1}{2 \pi i} \int_{c-i \infty}^{c+i \infty}\left(y^{s+1} / s(s+1)\right) f(s, k) d s .
$$

It is well known that $\zeta(s)=O\left(\theta^{-1}|s|^{\theta}\right)$, uniformly in $0<\theta \leq 1 / 2$ and $\operatorname{Re}(s)=1-\theta$. With $\theta=1 / 2 k$, it follows that for some fixed $C_{1}>1$, and uniformly in $k \geq 2, \operatorname{Re}(s)=2-1 / 2 k$,

$$
f(s, k)=O\left(C_{1}^{k} k^{k}|s|^{1 / 2}\right) \text {. }
$$


Although it is not essential to the proof, it will be convenient to have $C_{1}=4$. A little detail work, starting with the formula [11]

$$
\zeta(s)=s /(s-1)+s \sum_{n=1}^{\infty} \int_{0}^{1} u(n+u)^{-s-1} d u
$$

valid for $\operatorname{Re}(s)>0$, is now in order. For $n \leq|s|$ in the sum, one uses integration by parts, and with the obvious bounds for the other terms, this gives, for $s=1-\theta$,

$$
|\zeta(s)| \leq \theta^{-1}+\theta^{-1}\left(1+|s|^{\theta}\right)+(1 / 2)|s|^{\theta} /(1-\theta) .
$$

For $s>1,|\zeta(s)| \leq \zeta(\sigma)$, so with $\theta=1 / 2 k$, the claim that $C_{1}$ can be 4 holds provided

$$
4 k+2 k|s|^{\theta}+\left(\frac{1}{2-2 \theta}\right)|s|^{\theta} \leq 4 k \zeta(2-1 / 2 k)|s|^{\theta} .
$$

But $4 k \zeta(2-1 / 2 k)>4 k \zeta(2)+1$ since $\zeta^{\prime}(\sigma)<-1 / 4$ for $1<\sigma \leq 2$, so we just need

$$
4 \leq(4 \zeta(2)-2)|s|^{\theta} \text {. }
$$

The worst case is $k=2, s=3 / 4$, and even then $4 \leq 4.2619 \ldots$.

Now let $\Gamma$ be the linear path from $3-i \infty$ to $3+i \infty$, and let $\Gamma_{N, k}$ be the counterclockwise circuit of the rectangle with corners $3-i N$, $3+i N,(2-1 / 2 k)+i N$, and $(2-1 / 2 k)-i N$. Then

$$
\text { (31) } \begin{aligned}
\lim _{N \rightarrow \infty} & \left(\int_{\Gamma}-\int_{\Gamma_{N, k}}\right)\left(y^{s+1} f(s, k) / s(s+1)\right) d s \\
& =\int_{2-1 / 2 k-i \infty}^{2-1 / 2 k+i \infty}\left(y^{s+1} f(s, k) / s(s+1)\right) d s=E_{1}(y, k) \text {, say. }
\end{aligned}
$$

In view of $(30), E_{1}(y, k)=O\left(C_{1}^{k} k^{k} y^{3-1 / 2 k}\right)$. For $k^{2} \leq C_{1}^{-2} \log y$, a simple calculation now shows that

$$
E_{1}(y, k)=O\left(y^{3} \lambda^{k}(\log y)^{k-2} / k^{2}(k-1) !\right),
$$

which is the error allowed for in Lemma 2.

REMARK. The argument fails here without some hypothesis on $C_{1}$. This brings us to the kernel of the matter: we must evaluate the integral over $\Gamma_{N, k}$ to within $O\left(y^{3} \lambda^{k}(\log y)^{k-2} / k^{2}(k-1) !\right)$.

Let $\beta(s, k)=(s-2)^{k} f(s, k) / s(s+1)$. Then

$$
\begin{aligned}
& \frac{1}{2 \pi i} \int_{\Gamma_{N, k}}\left(y^{s+1} f(s, k) / s(s+1)\right) d s \\
& \quad=y^{3} \int_{\Gamma_{N, k}}\left((s-2)^{-k} \beta(s, k) y^{s-2} / 2 \pi i\right) d s,
\end{aligned}
$$


and the latter integral is, by the residue theroem, equal to the $(s-2)^{k-1}$ coefficient, say $T_{k-1}(y)$, in the Taylor series expansion of $\beta(s, k) y^{s-2}$ about 2 . To estimate this, we first note that for a complex analytic function $\xi$ on a disk of radius $r$, if $|\xi| \leq K$ on the disk, then by the Plancherel formula, $\left|\left(d^{j} / d s^{j}\right) \xi(s)\right| \leq K j ! r^{-j}$ at the center.

Now $(s-2) \zeta(s-2) / \zeta(s-1)=\lambda\left(1+a(s-2)+O(s-2)^{2}\right)$, uniformly in $|s-2| \leq 1 / 2$, say. Thus for arbitrary $j, 1 \leq j \leq k$, on the disk $|s-2| \leq j / 2 k$, we have

$$
\beta(s, k)=O\left(\lambda^{k} \exp (O(j))\right),
$$

so that from the observation above, if $D_{j}(k)=\left(d^{j} / d s^{j}\right) \beta(s, k)$ evaluated at $s=2$, then for $j \leq k$,

$$
D_{j}(k)=O\left((2 k / j)^{j} j ! \lambda^{k} \exp (O(j))\right) .
$$

(For $j=0$, we have $D_{j}(k)=\lambda^{k} / 6$, of course.) Now

$$
T_{k-1}(y)=((k-1) !)^{-1}\left(d^{k-1} / d s^{k-1}\right)\left(y^{s-2} \beta(s, k)\right),
$$

evaluated at $s=2$. Expanding the iterated derivative of a product as in the binomial theorem, we get

$$
(k-1) ! T_{k-1}(y)=\sum_{j=0}^{k-1}\left(\begin{array}{c}
k-1 \\
j
\end{array}\right)(\log y)^{k-1-j} D_{j}(k) .
$$

The main term here, corresponding to $j=0$, is $(1 / 6) \lambda^{k} \log ^{k-1} y$. For $j \geq 1$, we have, in the sum above,

$$
\begin{aligned}
& \left(\begin{array}{c}
k-1 \\
j
\end{array}\right)(\log y)^{k-1-j} D_{j}(k) \\
& \quad=O\left(\left(k^{j} / j !\right)(\log y)^{k-1-j}(2 k / j)^{j} j ! \lambda^{k} \exp (O(j))\right) \\
& \quad=(\log y)^{k-1} \cdot O_{\varepsilon}\left(k^{2 j}(\log y)^{-j}\left(j^{-(1-\varepsilon) j}\right)\right) .
\end{aligned}
$$

Thus, for $k^{2} \leq \log y$,

$$
T_{k-1}(y)=\left(\lambda^{k} \log ^{k-1} y /(k-1) !\right)\left(1+O\left(k^{2} / \log y\right)\right),
$$

which completes the proof of Lemma 2. With $C_{1}=4, C$ in Lemma 1 becomes $1 / 4$. We need another estimate for the case of large $k$.

LEMMA 3. For $k \geq 1$ and $y \geq 1$,

$$
A_{k}(y) \leq 4^{k+1} y^{2+3 / 2 \pi^{2}} \text {. }
$$


Proof. First note that this is trivial from the definition if $1 \leq y<4$, or if $k=1$. Now in (29), take $c=2+3 / 2 \pi^{2}$. From this, it follows that with $s=\left(2+3 / 2 \pi^{2}\right)+i \tau$,

$$
B_{k}(y) \leq\left(y^{c+1} / 2 \pi\right) \int_{-\infty}^{\infty}|f(s, k)| /|s(s+1)| d \tau .
$$

For $\sigma=\operatorname{Re}(s)>2$, by the product repesentation of the zeta function, and elementary properties of the linear fractional $\left(1+z p^{-\sigma}\right) /\left(1+z p^{1-\sigma}\right)$ on the circle $|z|=1$, we have

$$
|\zeta(s-1) / \zeta(s)| \leq|\zeta(\sigma-1) / \zeta(\sigma)| \text {. }
$$

Thus from $(38), B_{k}(y) \leq\left(\frac{1}{2 \pi}\right) y^{c+1}(\zeta(c-1) / \zeta(c))^{k} \cdot \pi / c$. Now taking $m=[y / 4]$ in $(26)$ gives

$$
A_{k}(y) \leq(3 / 5)(7 / 2)^{k} m^{-1}\left((y+m)^{c+1}-y^{c+1}\right) .
$$

Since this expression is increasing in $m / y$,

$$
A_{k}(y) \leq(3 / 5)(7 / 2)^{k} 4\left((5 / 4)^{c+1}-1\right) y^{c},
$$

which for $k \geq 2$ is $\leq 3 \cdot 4^{k} y^{c}<4^{k+1} y^{c}$. This proves Lemma 3 .

4. Estimation of $W_{l}(x, N)$. From (14), (15) and Lemma 1, we have

(40) $W_{l}(x, N) \leq \frac{x^{2} \lambda^{l+1} 2^{l+1}}{2(l !)}$

$$
\begin{aligned}
& \sum_{\substack{k_{1} k_{2} \cdots k_{l} \leq x \exp \left(-16(l+1)^{2}\right) \\
k_{i}>N \text { for } 1 \leq i \leq l}} k_{1}^{-2} k_{2}^{-2} \cdots k_{l}^{-2}\left(\log x-\sum_{1}^{l} \log k_{i}\right)^{l} \\
& \cdot\left(1+O\left(l^{2} /\left(\log x-\sum_{1}^{l} \log k_{i}\right)\right)\right) \\
& +2^{l+1} \quad \sum \quad A_{l+1}\left(x / k_{1} k_{2} \cdots k_{l}\right) \text {. } \\
& x \exp \left(-16(l+1)^{2}\right)<k_{1} k_{2} \cdots k_{l} \leq x \\
& k_{l}>N \text { for } 1 \leq i \leq l
\end{aligned}
$$

In the second term here, $u=\log x-\sum_{1}^{l} \log k_{i}<16(l+1)^{2}$, so that Lemma 1 is not applicable. Happily, for this term there is no need of 
sharp estimates. We get a crude, but adequate, bound from

LEMMA 4. For $l \geq 1, N \geq 8$ and $x \geq(N+1)^{l} e^{16 l^{2}}$,

$$
\begin{aligned}
& 2^{l+1} \sum_{\substack{x \exp \left(-16(l+1)^{2}\right)<k_{1} k_{2} \cdots k_{l} \leq x \\
k_{1}>N \text { for } 1 \leq i \leq l}} A_{l+1}\left(x / k_{1} k_{2} \cdots k_{l}\right) \\
& \ll\left((16)^{l} x \exp \left(16 l^{2}\left(1+3 /\left(2 \pi^{2}\right)\right)\right)(\log x)^{l} / l !\right) .
\end{aligned}
$$

The application of the lemma will be to cases in which $N \leq(\log x)^{2}$ and $l \leq(\log x)^{1 / 3}$, so that the upper bound given in Lemma 4 comes to $O_{\varepsilon}\left(x^{1+\varepsilon}\right)$, or what is good enough for our purposes, to $O\left(x^{3 / 2}\right)$.

To prove Lemma 4, we first note that from Lemma 3,

$$
A_{l+1}(y) \leq 4^{l+2} y^{2+\lambda / 4}
$$

Thus

$$
\begin{aligned}
& 2^{l+1} \sum_{\substack{x \exp \left(-16(l+1)^{2}\right)<k_{1} k_{2} \cdots k_{l} \leq x \\
k_{i}>N \text { for } 1 \leq i \leq l}} A_{l+1}\left(x / k_{1} k_{2} \cdots k_{l}\right) \\
& \leq(16)^{l+1} x^{2} \sum_{\text {(same range) }} k_{1}^{-2} \cdots k_{l}^{-2} \exp \left(\frac{\lambda}{4}\left(\log x-\sum_{1}^{l} \log k_{i}\right)\right) \\
& \leq(16)^{l+1} x^{2+\lambda / 4} \sum_{\text {all } k_{l}>N, \prod_{1}^{l} k_{l}>x \exp \left(-16(l+1)^{2}\right)} \prod_{1}^{l} k_{i}^{-(2+\lambda / 4)} \text {. }
\end{aligned}
$$

The sum in the right side of (42) above is itself

$$
\leq \iint \cdots \int_{R} \prod_{i=1}^{l} t_{i}^{-(2+\lambda / 4)} d t_{l} \cdots d t_{1},
$$

where $R=\left\{\left(t_{1}, t_{2}, \ldots, t_{l}\right): t_{i} \geq N\right.$ for $1 \leq i \leq l$ and $\prod_{1}^{l} t_{i} \geq$ $\left.x e^{-16 l^{2}}\right\}$.

On setting $s_{i}=\log t_{i}, 1 \leq i \leq l$, this integral becomes

$$
\iint \cdots \int_{R_{l}^{\prime}} \exp \left(-\beta \sum_{i=1}^{l} s_{i}\right) d s_{l} \cdots d s_{1},
$$


where $\beta=1+\lambda / 4$, and where

$$
\begin{aligned}
& R_{l}^{\prime}=\left\{\left(s_{1}, \ldots, s_{l}\right): s_{i} \geq \log N \text { for } 1 \leq i \leq l\right. \\
& \left.\quad \text { and } \sum_{1}^{l} s_{i} \geq \log x-16 l^{2}\right\} .
\end{aligned}
$$

Seen as an iterated integral, the innermost integral is a function of $s_{1}, s_{2}, \ldots, s_{l-1}$ and is

$$
\begin{aligned}
& \int_{\max \left(\log x-16 l^{2}-s_{1}-s_{2}-\cdots-s_{l-1}, \log N\right)}^{\infty} \exp \left(-\beta\left(s_{1}+s_{2}+\cdots+s_{l-1}\right)\right) e^{-\beta s_{l}} d s_{l} \\
& \quad \leq \min \left\{x^{-\beta} e^{16 \beta l^{2}}, N^{-\beta} e^{-\beta\left(s_{1}+s_{2}+\cdots+s_{l-1}\right)}\right\} .
\end{aligned}
$$

Thus the original multiple integral is

$$
\begin{aligned}
& \leq x^{-\beta} e^{16 \beta l^{2}} \operatorname{Vol}\left(\left\{\left(s_{1}, s_{2}, \ldots, s_{l-1}\right): \log x-16 l^{2}-\log N\right.\right. \\
& \left.\left.\quad>\sum_{1}^{l-1} s_{i} \text { and all } s_{i}<\log N\right\}\right) \\
& \quad+N^{-\beta} \iint \cdots \int_{R_{l-1}^{\prime}} e^{-\beta\left(s_{1}+s_{2}+\cdots+s_{l-1}\right)} d s_{l-1}, \cdots d s_{1},
\end{aligned}
$$

where $R_{l-1}^{\prime}:=\left\{\left(s_{1}, s_{2}, \ldots, s_{l-1}\right): s_{i} \geq \log N\right.$ and $\sum_{1}^{l-1} s_{i} \geq \log x-$ $\left.16 l^{2}-\log N\right\}$. The first term above is just $x^{-\beta} \exp \left(16 \beta l^{2}\right)(\log x)^{l} / l !$, while the second term is of the same form as the original integral. Hence, we proceed by induction. Let

$$
F(l, z):=\iint \cdots \int_{R(l, z)} \exp \left(-\beta \sum_{i=1}^{l} s_{i}\right) d s_{l} \cdots d s_{1},
$$

where $R(l, z):=\left\{\left(s_{1}, \ldots, s_{l}\right): s_{i} \geq \log N\right.$ for $1 \leq i \leq l$ and $\sum_{i=1}^{l} s_{i}$ $\geq z\}$. In this terminology, we have shown above that

$$
F(l, z) \leq e^{-\beta z}\left(z+16 l^{2}\right)^{l} / l !+N^{-\beta} F(l-1, z-\log N) .
$$

Now $F(1, z)=\int_{\max (\log N, z)}^{\infty} e^{-\beta s} d s=\beta^{-1} \min \left(N^{-\beta}, e^{-\beta z}\right)$, and in particular if $z>\log N$ then $F(1, z)=\beta^{-1} e^{-\beta z}$. Now from this and from (43), if $z>l \log N$ then

$$
F(l, z) \leq e^{-\beta z} \sum_{j=2}^{l}\left(z+16 j^{2}\right)^{j} N^{\beta(j-L)} / j !
$$


Since $z>\log N>2 l$ under the assumption $N \geq 8$ in Lemma 4, the sum in (44) is dominated by the last term $\left(z+16 l^{2}\right)^{l} / l$ !, so that

$$
F(l, z) \ll e^{-\beta z}\left(\left(z+16 l^{2}\right)^{l} / l !\right) .
$$

We apply (45) with $z=\log x=16 l^{2}$ to obtain, for $x>N^{l} e^{16 l^{2}}$,

$$
\iint \cdots \int_{R} \prod_{i=1}^{l} t_{i}^{-(2+\lambda / 4)} d t_{l} \cdots d t_{1} \ll x^{-\beta} \exp \left(16 l^{2} \beta\right)\left((\log x)^{l} / l !\right) .
$$

In view of (42) and the following inequalty, this proves Lemma 4.

For $x$ sufficiently large, though, if $l \leq(\log x)^{1 / 3}$ and $N \leq(\log x)^{2}$, then

$$
(16)^{l} x \exp \left(16 \beta l^{2}\right)\left(N^{\beta}+(\log x)^{l} / l !\right)<x^{3 / 2} .
$$

Thus for large $x$ the second term in (40) is negligible, even in comparison to the potential error in the first term of (40). The main term of that, putting aside for now the contribution from the " $O$ " in $\left(1+O\left(l^{2} /\left(\log x-\sum_{1}^{l} \log k_{i}\right)\right)\right)$, is

$$
\sum_{\substack{k_{1} k_{2} \cdots k_{l} \leq x \exp \left(-16(l+1)^{2}\right) \\ k_{l}>N \text { for } 1 \leq i \leq l}} k_{1}^{-2} k_{2}^{-2} \cdots k_{l}^{-2}\left(\log x-\sum_{1}^{l} \log \left(k_{i}\right)\right)^{l} .
$$

But this is less than

$$
\begin{gathered}
\int_{t_{1} t_{2} \cdots t_{l} \leq x \exp \left(-16 l^{2}\right)} \int \cdots \int\left(t_{1}^{-2} t_{2}^{-2} \cdots t_{l}^{-2}\right)(\log x)^{l} d t_{l} \cdots d t_{2} d t_{1} \\
t_{i} \geq N \text { for } 1 \leq i \leq l \\
\leq(\log x / N)^{l} .
\end{gathered}
$$

The error term just put aside is likewise

$\ll l^{2} \iint \cdots \int\left(t_{1}^{-2} \cdots t_{l}^{-2}\right)(\log x)^{l-1} d t_{l} \cdots d t_{1} \ll\left(l^{2} / \log x\right)(\log x / N)^{t_{3}}$

Thus for $x$ sufficiently large, $l \leq(\log x)^{1 / 3}$ and $N \leq(\log x)^{2}$,

(48) $W_{l}(x, N) \leq\left(1+O\left(l^{2} / \log x\right)\right)(\log x / N)^{l}\left(2^{l} \lambda^{l+1} / l !\right) x^{2}+O\left(x^{3 / 2}\right)$. 
Next we obtain a similar lower bound for $W_{l}(x, N)$. From (17) and Lemma 1, we have

$$
\begin{gathered}
W_{l}(x, N) \geq\left(x^{2} 2^{l} \lambda^{l+1} / l !\right) \\
\sum_{k_{1} k_{2} \cdots k_{l} \leq x \exp \left(-16(l+1)^{2}\right)(1+1 / N)^{-2 l}}^{\substack{k_{l}>N \text { for } 1 \leq i \leq l \\
1}} k_{1}^{-2} k_{2}^{-2} \cdots k_{l}^{-2} \\
\cdot\left(\log x-\sum_{i=1}^{l} \log \left((1+1 / N)^{2} k_{i}\right)\right)^{l}+\text { two error terms. }
\end{gathered}
$$

Let $x^{\prime}=x(1+1 / N)^{-2 l}$, and let $S_{l}:=\left\{K=\left(k_{1}, k_{2}, \ldots, k_{l}\right)\right.$ : $k_{1} k_{2} \cdots k_{l} \leq x^{\prime} \exp \left(-16(l+1)^{2}\right)$ and $k_{i}>N$ for $\left.1 \leq i \leq l\right\}$. The first of the above-mentioned error terms stems from the factor $1+$ $O\left(k^{2} / \log y\right)$ in Lemma 1 . For $K \in S_{l}$, this factor, applied to each of the contributions to the sum in (49), is $1+O\left((l+1)^{2} / \log x\right)$ so that the whole sum is also perturbed by only a factor of $\left(1+O\left((l+1)^{2} / \log x\right)\right.$ due to that source of error. The other term in (49) is the contribution to $\sum \sum \cdots \sum A_{l+1}\left(x^{\prime} / k_{1} k_{2} \cdots k_{l}\right)$ due to $K=\left(k_{1}, k_{2}, \ldots, k_{l}\right)$ for which $k_{i}>N, 1 \leq i \leq l$, but $\prod_{i=1}^{l} k_{i}>$ $x^{\prime} \exp \left(-16(l+1)^{2}\right)$.

For $x$ sufficiently large, if $N \leq \log ^{2} x$ and $l \leq \log ^{1 / 3} x$, then the hypotheses of Lemma 4 are satisfied, so that this error term is $O\left((16)^{l} x^{\prime} e^{16 l^{2} \beta}\left(\left(\log x^{\prime}\right)^{l} / l !\right)\right)$ and thus $O\left(x^{3 / 2}\right)$ as before. Hence, for such $x, N$ and $l$,

$$
W_{l}(x, N) \geq\left(2^{l}\left(x^{\prime}\right)^{2} \lambda^{l+1} / l !\right)\left(1+O\left((l+1)^{2} / \log x\right) P+O\left(x^{3 / 2}\right),\right.
$$

where

$$
P=\sum_{\substack{k_{1} k_{2} \cdots k_{l} \leq x^{\prime} \exp \left(-16(l+1)^{2}\right) \\ k_{i}>N \text { for } 1 \leq i \leq l}} k_{1}^{-2} k_{2}^{-2} \cdots k_{l}^{-2}\left(\log \left(x^{\prime}\right)-\sum_{i=1}^{l} \log k_{i}\right)^{l} .
$$

Now we need a lower bound for $P$. Clearly,

$$
P \geq \int_{R} t_{1}^{-2} t_{2}^{-2} \cdots t_{l}^{-2}\left(\log \left(x^{\prime}\right)-\sum_{i=1}^{l} \log t_{i}\right)^{l} d t_{l} \cdots d t_{1},
$$

where $R=\left\{\left(t_{1}, t_{2}, \ldots t_{l}\right): t_{1} t_{2} \cdots t_{l} \leq x^{\prime} e^{-16(l+1)^{2}}\right.$ and $t_{i} \geq N+1$ for $1 \leq i \leq l\}$. After a change of variables $\left(u_{i}=\log t_{i}-\log (N+1)\right.$, 
$1 \leq i \leq l)$ this integral becomes

$$
\begin{aligned}
& (N+1)^{-l} \int_{U} e^{-\left(u_{1}+u_{2}+\cdots+u_{l}\right)} \\
& \quad \cdot\left(\log \left(x^{\prime}\right)-l \log (N+1)-\sum_{i=1}^{l} u_{i}\right)^{l} d u_{l} \cdots d u_{1},
\end{aligned}
$$

where $U=\left\{\left(u_{1}, u_{2}, \ldots, u_{l}\right): \sum_{i=1}^{l} u_{i} \leq \log \left(x^{\prime}\right)-16(l+1)^{2}-\right.$ $l \log (N+1)$ and $u_{i} \geq 0$ for $\left.1 \leq i \leq l\right\}$. This, though, is just

$$
(N+1)^{-l} \int_{0}^{L}\left(u^{l-1} /(l-1) !\right) e^{-u}(M-u)^{l} d u,
$$

where $L=\log \left(x^{\prime}\right)=l \log (N+1)-16(l+1)^{2}$ and $M=\log \left(x^{\prime}\right)-$ $l \log (N+1)$. Thus

$$
P \geq\left((N+1)^{-l} /(l-1) !\right)\left\{\int_{0}^{\infty}-\int_{L}^{\infty}\left(u^{l-1} e^{-u}(M-u)^{l} d u\right\}\right.
$$

The $-\int_{L}^{\infty}$ contribution here is quite small. In fact, for large $x$, for $N \leq \log ^{2} x$ and for $l \leq(\log x)^{1 / 3}$,

$$
\int_{L}^{\infty} u^{l-1}(M-u)^{l} e^{-u} d u \leq \int_{L}^{\infty} u^{2 l-1} e^{-u} d u \leq 2^{2 l-1} e^{-L},
$$

this last because $(1+1 / u)^{2 l-1} e^{-1}<\frac{1}{2}$ throughout the interval of integration. But in view of the constraints on $l$ and $N, 2^{2 l-1} e^{-L} \leq$ $x^{-3 / 4}$ for large $x$. The main term in our lower bound for $P$ is

$$
\begin{aligned}
\left((N+1)^{-l} /(l-1) !\right) & \int_{0}^{\infty} u^{l-1} e^{-u}(M-u)^{l} d u \\
& =\left((N+1)^{-l} /(l-1) !\right) \sum_{j=0}^{l}(-1)^{j}\left(\begin{array}{l}
l \\
j
\end{array}\right) M^{l-j} \int_{0}^{\infty} u^{l-1+j} e^{-u} d u \\
& =(N+1)^{-l} M^{l} \sum_{j=0}^{l}(-M)^{-j} l(l-1+j) ! /((j !)(l-j) !) .
\end{aligned}
$$

In view of the constraints on $l$ and $N$, we have $M \geq(1-\varepsilon) \log x$. ( $\varepsilon$ may be taken as small as we please by choosing a large enough lower bound for $x$ ). Thus the last sum above is dominated by its first term, and it simplifies to $\left(1+O\left(l^{2} / \log x\right)\right)$. Thus

$$
P=\left(1+O\left(l^{2} / \log x\right)\right)(N+1)^{-l} M^{l} .
$$


From (50), we now conclude that

$$
\begin{aligned}
W_{l}(x, N) \geq & \left(2^{l}\left(x^{\prime}\right)^{2} \lambda^{l+1} / l !\right)\left(1+O\left((l+1)^{2} / \log x\right)\right)(N+1)^{-l} \\
& \cdot\left(\log \left(x^{\prime}\right)-l \log (N+1)\right)^{l}+O\left(x^{3 / 2}\right) .
\end{aligned}
$$

Since $x^{\prime}=(1+1 / N)^{-2 l} x$, this boils down to

$$
\begin{aligned}
W_{l}(x, N) \geq & \left(2^{l} x^{2} \lambda^{l+1}(\log x)^{l} / N^{l}(l !)\right)(1+1 / N)^{-3 l} \\
& \times\left(1+O\left(l^{2} \log \log x / \log x\right)\right)+O\left(x^{3 / 2}\right),
\end{aligned}
$$

for $1 \leq l \leq(\log x)^{1 / 3}, N \leq(\log x)^{2}$ and $x$ sufficiently large. Together with (48), and under the same constraints, this gives

$$
\begin{aligned}
W_{l}(x, N)= & \frac{x^{2} 2^{l} \lambda^{l+1}}{(l !)}\left(\frac{\log x}{N}\right)^{l} \\
& \times \exp \left(O\left(\frac{l^{2} \log \log x}{\log x}\right)+O\left(\frac{l}{N}\right)\right)+O\left(x^{3 / 2}\right) .
\end{aligned}
$$

Now from (13),

$$
\Phi^{ \pm}(x, \alpha)=\frac{1}{2} \sum_{l=0}^{\infty}(-1)^{l} W_{l}\left(x, N^{ \pm}\right),
$$

with $N^{+}=[\alpha \log x]$ for $\Phi^{+}$and $N^{-}=[\alpha \log x]-1$ for $\Phi^{-}$. From (12), if we truncate this sum we get lower and upper bounds: if $A$ is odd and $B=A+1$, then

$$
\frac{1}{2} \sum_{l=0}^{A}(-1)^{l} W_{l}\left(X, N^{-}\right)<\Phi(x, \alpha)<\frac{1}{2} \sum_{l=0}^{B}(-1)^{l} W_{l}\left(x, N^{+}\right) .
$$

If we choose $B=\left[(\log x)^{1 / 3}\right]$, then for $\alpha>4 / \log \log x$ and $l \leq B$, the $l^{2} \log \log x / \log x$ contribution to the error factor in (55) dominates that from $l / N$, and both are small, so that (55) boils down to

$$
\begin{aligned}
W_{l}\left(x, N^{ \pm}\right)= & \frac{x^{2} 2^{l} \lambda^{l+1}}{(l !)}\left(\frac{\log x}{N^{ \pm}}\right)^{l}\left(1+O\left(\frac{l^{2} \log \log x}{\log x}\right)\right) \\
& +O\left(x^{3 / 2}\right)
\end{aligned}
$$

for such $l, N$, and $x$. Thus in (56), the main terms are

$$
\begin{aligned}
& \frac{1}{2} \sum_{l=0}^{A} x^{2} 2^{l} \lambda^{l+1}\left(-\log x / N^{-}\right)^{l} / l ! \quad \text { and } \\
& \frac{1}{2} \sum_{l=0}^{B} x^{2} 2^{l} \lambda^{l+1}\left(-\log x / N^{+}\right)^{l} / l !
\end{aligned}
$$


and the error factor in (57) perturbs these by at most

$$
O\left(x^{2} \sum_{l=0}^{\infty} 2^{l} \lambda^{l+1}\left(\log x / N^{-}\right)^{l}\left(l^{2} \log \log x / \log x\right) / l !\right) .
$$

But this is $O\left(\left(x^{2} \log \log x / \log x\right)\left(z^{2}+1\right) e^{z}\right)$, where $z=2 \lambda \log x / N^{-}$. Now for $\alpha \geq 4 / \log \log x, N^{-} \geq(4 \log x / \log \log x)-2$ so that $z \leq$ $\frac{1}{2} \lambda \log \log x$, and

$\left(x^{2} \log \log x / \log x\right)\left(z^{2}+1\right) e^{z} \ll x^{2}\left(\log \log x\left(1+\alpha^{-2}\right) / \log x\right) e^{2 \lambda / \alpha}$.

This brings us to the main terms in (56). They are

$$
\frac{1}{2} \lambda x^{2} \sum_{l=0}^{A}\left(\frac{2 \lambda \log x}{N^{-}}\right)^{l} / l !, \quad \text { and } \frac{1}{2} \lambda x^{2} \sum_{l=0}^{B}\left(\frac{2 \lambda \log x}{N^{+}}\right)^{l} / l !
$$

respectively. If we replace $A$ and $B$ with $\infty$ in these sums, the resulting change is $O\left((2 \lambda / \alpha)^{B} / B !\right)$, and with $B=\left[\log x^{1 / 3}\right]$, that is $\ll 1 / \log x$. Thus the main terms above are

$$
\frac{1}{2} \lambda x^{2}\left(\exp \left(-2 \lambda \log x / N^{ \pm}\right)+O(1 / \log x)\right) .
$$

Replacing $\left(\log x / N^{ \pm}\right)$with $1 / \alpha$ here introduces an error factor of $\exp \left(O\left(1 / \alpha^{2} \log x\right)\right)$, so that the main terms boil down to

$$
\frac{1}{2} \lambda x^{2} e^{-2 \lambda / \alpha}\left(1+O\left(\left(1+\alpha^{-2}\right) / \log x\right)\right)
$$

That is,

$$
\begin{aligned}
&(58) \Phi(x, \alpha)= \frac{1}{2} \lambda x^{2} e^{-2 \lambda / \alpha}\left(1+O\left(1 / \alpha^{2} \log x\right)+O(1 / \log x)\right) \\
&+O\left(x^{2} \log \log x\left(1+\alpha^{-2}\right) / \log x\right) e^{2 \lambda / \alpha}+O\left(x^{3 / 2}\right) \\
&=\frac{1}{2} \lambda x^{2} e^{-2 \lambda / \alpha}\left(1+O\left(e^{4 \lambda / \alpha} \log \log x\left(1+\alpha^{-2}\right)(\log x)^{-1}\right)\right),
\end{aligned}
$$

for all sufficiently large $x$ and all $\alpha, 4 / \log \log x \leq \alpha \leq(\log x)^{2}$. The condition $N \leq(\log x)^{2}$, which roughly coincides with $\alpha \leq \log x$, has been necessary in the workings of the main argument. But for $\alpha \geq \log x$, the claim made by Theorem 1 reduces to an assertion that $\Phi(x, \alpha)=\frac{1}{2} \lambda x^{2}(1+O(\log \log x / \log x))$. Now $\Phi(x, \alpha)$ is a nondecreasing function of $\alpha$. But the upper bound part of this follows from Lemma 1, while the required lower bound follows from what we have proved above, on taking $\alpha=\log x$. Thus the theorem, while of no interest in this case, happens nonetheless to hold. 


\section{REFERENCES}

[1] R. Bumby, Hausdorf dimension of sets arising in number theory, Lecture Notes in Mathematics, 1135 (1-8) New York Number Theory Seminar, Springer NY 1985.

[2] T. W. Cusick, Continuants with bounded digits, Mathematika, 24 (1977), 166172.

[3] _ Continuants with bounded digits II, Mathematika, 25 (1978), 107-109.

[4] J. Galambos, The distribution of the largest coefficient in continued fraction expansions, Quart. J. Math. Oxford Ser. (2), 23 (1972), 147-151.

[5] _. The largest coefficient in continued fractions and related problems, Diophantine approximation and its applications (Proc. Conf. Washington D. C. 1972) 101-109, Academic Press, NY 1973.

[6] I. J. Good, The fractional dimension theory of continued fractions, Proc. Cambridge Phil. Soc., 37 (1941), 199-228.

[7] D. Hensley, A truncated Gauss-Kuzmin law, Trans. Amer. Math. Soc., 306 (1) 1988.

[8] _ - The distribution of badly approximable numbers and continuants with bounded digits, Proc. Int. Conference on Number Theory, Quebec 1987 (371385).

[9] _ - The distribution of badly approximable numbers and continuants with bounded digits, II, J. Number Theory, 34 (1990), 293-334.

[10] _ Continued fraction Cantor sets, Hausdorff dimension, and functional analysis, J. Number Theory, to appear.

[11] Aleksandr Ivic, The Riemann Zeta Fucntion, Chapter 1, Wiley, NY 1985.

Received March 7, 1990 and in revised form October 25, 1990.

TeXas A \& M University

College Station, TX 77843-3368 



\section{PACIFIC JOURNAL OF MATHEMATICS EDITORS}

\author{
V. S. VARADARAJAN \\ (Managing Editor) \\ University of California \\ Los Angeles, CA 90024-1555-05 \\ Herbert Clemens \\ University of Utah \\ Salt Lake City, UT 84112 \\ THOMAS ENRIGHT \\ University of California, San Diego \\ La Jolla, CA 92093
}

Nicholas ERcolani

University of Arizona

Tucson, AZ 85721

R. FINN

Stanford University

Stanford, CA 94305

VAUGHAN F. R. JONES

University of California

Berkeley, CA 94720

STEVEN KeRCKHOFF

Stanford University

Stanford, CA 94305

\section{C. MOORE \\ University of California \\ Berkeley, CA 94720}

MARTIN SChaRLEMANN

University of California

Santa Barbara, CA 93106

HAROLd STARK

University of California, San Diego

La Jolla, CA 92093

\section{ASSOCIATE EDITORS}

\begin{tabular}{|c|c|c|c|c|}
\hline R. ARENS & $\begin{array}{l}\text { E. F. BECKENBACH } \\
(1906-1982)\end{array}$ & NeumanN & $\begin{array}{l}\text { F. WoLF } \\
(1904-1989)\end{array}$ & K. YoshidA \\
\hline \multicolumn{5}{|c|}{ SUPPORTING INSTITUTIONS } \\
\hline \multirow{2}{*}{\multicolumn{2}{|c|}{$\begin{array}{l}\text { IVERSITY OF ARIZONA } \\
\text { IVERSITY OF BRITISH COLUMBIA }\end{array}$}} & UNIVERS & Y OF OREGON & \\
\hline & & UNIVERS & OF SOUTHER & RNIA \\
\hline \multicolumn{2}{|c|}{ IFORNIA INSTITUTE OF TECHNOLOGY } & STANFOI & UNIVERSITY & \\
\hline \multirow{2}{*}{\multicolumn{2}{|c|}{$\begin{array}{l}\text { IVERSITY OF CALIFORNIA } \\
\text { NTANA STATE UNIVERSITY }\end{array}$}} & UNIVERS & OF HAWAII & \\
\hline \multirow{2}{*}{\multicolumn{2}{|c|}{$\begin{array}{l}\text { NTANA STATE UNIVERSITY } \\
\text { IVERSITY OF NEVADA, RENO }\end{array}$}} & UNIVERS & Y OF TOKYO & \\
\hline & & UNIVERS & OF UTAH & \\
\hline \multirow{2}{*}{\multicolumn{2}{|c|}{$\begin{array}{l}\text { N MEXICO STATE UNIVERSITY } \\
\text { EGON STATE UNIVERSITY }\end{array}$}} & WASHING & N STATE UNI & RSITY \\
\hline & & UNIVERS & OF WASHING & \\
\hline
\end{tabular}

The Supporting Institutions listed above contribute to the cost of publication of this Journal, but they are not owners or publishers and have no responsibility for its content or policies.

Mathematical papers intended for publication in the Pacific Journal of Mathematics should be in typed form or offset-reproduced (not dittoed), double spaced with large margins. Please do not use built up fractions in the text of the manuscript. However, you may use them in the displayed equations. Underline Greek letters in red, German in green, and script in blue. The first paragraph must be capable of being used separately as a synopsis of the entire paper. In particular it should contain no bibliographic references. Please propose a heading for the odd numbered pages of less than 35 characters. Manuscripts, in triplicate, may be sent to any one of the editors. Please classify according to the 1991 Mathematics Subject Classification scheme which can be found in the December index volumes of Mathematical Reviews. Supply name and address of author to whom proofs should be sent. All other communications should be addressed to the managing editor, or Elaine Barth, University of California, Los Angeles, California 90024-1555-05.

There are page-charges associated with articles appearing in the Pacific Journal of Mathematics. These charges are expected to be paid by the author's University, Government Agency or Company. If the author or authors do not have access to such Institutional support these charges are waived. Single authors will receive 50 free reprints; joint authors will receive a total of 100 free reprints. Additional copies may be obtained at cost in multiples of 50 .

The Pacific Journal of Mathematics (ISSN 0030-8730) is published monthly except for July and August. Regular subscription rate: $\$ 190.00$ a year (10 issues). Special rate: $\$ 95.00$ a year to individual members of supporting institutions.

Subscriptions, orders for numbers issued in the last three calendar years, and changes of address should be sent to Pacific Journal of Mathematics, P.O. Box 969, Carmel Valley, CA 93924, U.S.A. Old back numbers obtainable from Kraus Periodicals Co., Route 100, Millwood, NY 10546.

The Pacific Journal of Mathematics at P.O. Box 969, Carmel Valley, CA 93924 (ISSN 0030-8730) is published monthly except for July and August. Second-class postage paid at Carmel Valley, Californiä 93924, and additional mailing offices. Postmaster: send address changes to Pacific Journal of Mathematics, P.O. Box 969, Carmel Valley, CA 93924.

\section{PUBLISHED BY PACIFIC JOURNAL OF MATHEMATICS, A NON-PROFIT CORPORATION}




\section{Pacific Journal of Mathematics}

\section{Vol. 151, No. $2 \quad$ December, 1991}

Michael G. Eastwood and A. M. Pilato, On the density of twistor

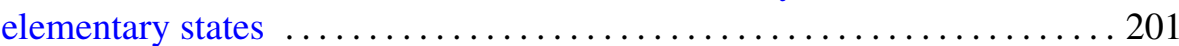

Brian E. Forrest, Arens regularity and discrete groups $\ldots \ldots \ldots \ldots \ldots \ldots 217$

Yu Li Fu, On Lipschitz stability for F.D.E ..................... 229

Douglas Austin Hensley, The largest digit in the continued fraction expansion of a rational number $\ldots \ldots \ldots \ldots \ldots \ldots \ldots \ldots \ldots \ldots \ldots . \ldots 237$

Uwe Kaiser, Link homotopy in $\mathbb{R}^{3}$ and $S^{3}$

Ronald Leslie Lipsman, The Penney-Fujiwara Plancherel formula for abelian symmetric spaces and completely solvable homogeneous

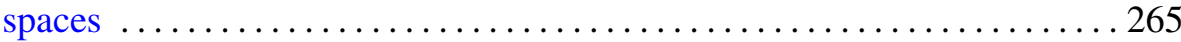

Florin G. Radulescu, Singularity of the radial subalgebra of $\mathscr{L}\left(F_{N}\right)$ and the

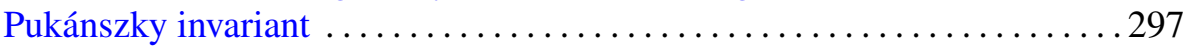

Albert Jeu-Liang Sheu, The structure of twisted SU(3) groups $\ldots . \ldots \ldots . \ldots 307$

Morwen Thistlethwaite, On the algebraic part of an alternating link . . . . . 317

Thomas (Toma) V. Tonev, Multi-tuple hulls .................. 335

Arno van den Essen, A note on Meisters and Olech's proof of the global asymptotic stability Jacobian conjecture ..................... 351

Hendrik J. van Maldeghem, A characterization of the finite Moufang hexagons by generalized homologies

Bun Wong, A note on homotopy complex surfaces with negative tangent bundles

Chung-Tao Yang, Any Blaschke manifold of the homotopy type of $\mathbf{C} P^{n}$ has the right volume 